\section{Stress im Job: Kein Risikofaktor für Krebs}

\begin{abstract}
Berufsstress, durch hohe Anforderungen und/oder wenig Handlungspielraum , geht nicht mit einem höheren Krebsrisiko einher. Dies ergab die Metaanalyse zwölf europäischer Kohortenstudien mit rund 116.000 Probanden, von welchen $5,0 \%$ im Beobachtungszeitraum an Krebs erkrankten. Es ergaben sich keine Beweise dafür, dass die Belastung im Job das Gesamtkrebsrisiko oder die Gefahr, an bestimmten Tumorentitäten zu erkranken, erhöht.
\end{abstract}

Heikkilä Ket al, BMJ 2013; doi: 10.113/bmj.f165

\section{Neurodermitiker dürfen nicht rein!}

Immer häufiger dienen Fingerabdrücke zur Identifizierung, z. B. am Computer oder zur Eingangskontrolle. Wer unter einem Handekzem leidet, bekommt da leicht Probleme: In einer Studie scheiterten $27 \%$ der Handdermatitis-Patienten, aber nur 2\% der gesunden Kontrollen an einem biometrischen Sicherheitssystem, meist wegen abnormer weißer Linien oder einer Dystrophie des Fingerbeerenmusters.

Kek Lee C et al, Arch Dermatol 2012 (online first)

\section{Ayurveda so gut wie Coxib}

Ayurveda scheint bei Arthrose ähnlich wirksam zu sein wie Glucosaminsulfat und Celecoxib. Indische Rheumatologen verglichen die Präparate bei 440 Patienten: Diese erhielten sechs Monate lang täglich entweder $200 \mathrm{mg}$ Celecoxib, $2 \mathrm{~g}$ Glucosaminsulfat, den Extrakt SGC (Mondsamengewächs Guduchi, Ingwer, Amalakifrucht) oder SGCG, der zusätzlich Weihrauch enthält. Es gab keinen Wirkunterschied bei Schmerz und Gelenkfunktion. Allerdings gab es bei den Pflanzenpräparaten mehr Studienabbrecher wegen Nebenwirkungen.

Chopra A et al, Rheumatology 2013 (online first)

Lässt Darwin grüßen?

\title{
Hänflinge müssen früher sterben
}

Männer, die als Jugendliche schwach und ohne Muskelkraft sind, setzen ihrem Leben häufiger selbst ein Ende oder versterben früher an den Folgen von Herzund Kreislauf-Leiden als kräftigere Geschlechtsgenossen.

Das ergab eine prospektive Kohortenstudie. In dieser führten schwedische Forscher mit über 1,1 Millionen Jugendlichen zwischen 16 und 19 Jahren verschiedene Tests zur Muskelstärke durch, maßen Blutdruck und bestimmten den BodyMass-Index (BMI). Anschließend wurde die Gesundheit der Probanden über durchschnittlich 24 Jahre verfolgt. Im Studienzeitraum verstarben 26.145 Männer. Haupttodesursache war mit 25,9\% ein Unfall, gefolgt von Suizid (22,3\%), Krebs $(14,9 \%)$ und kardiovaskulären Erkrankun- gen (7,8\%). Als unabhängige Risikofaktoren für einen frühen Tod erwiesen sich der BMI, der diastolische Blutdruck sowie die Stärke der Muskelkraft. Männer, die bei den Krafttests punkten konnten, hatten unabhängig von Blutdruck und BMI innerhalb des Studienzeitraums eine um $35 \%$ niedrigere kardiovaskuläre Sterblichkeit als die Teilnehmer der Referenzgruppe. Zudem hatten die Stärksten um 20-30\% niedrigere Suizidraten und ein 15-65\% geringeres Risiko, eine psychische Erkrankung zu entwickeln. Als zuverlässigstes Instrument hat sich der sogenannte Handgrip-Strength-Test erwiesen. Die Autoren halten ihn in der Prävention für hilfreich, um Jugendlichen mit geringer Muskelkraft frühzeitig zu einer Verbesserung ihrer körperlichen Fitness zu raten. (st)

Ortega FB et al, BMJ 2012, 345: e7279

\section{Erfolgreicher als SSRI}

\section{Strom am Schädel lindert Depression}

Per Gleichstromtherapie lassen sich Depressionen so gut lindern wie mit einem SSRI. Noch mehr steigt die Stimmung, wenn man beide Verfahren kombiniert.

Als Alternative zu Antidepressiva wird die transkranielle Gleichstromstimulation (tDCS) getestet. Forscher um Dr. Andre Brunoni aus São Paulo, Brasilien, konnten bei relativ schwer erkrankten Patienten 30 Punkte auf der Montgomery-AsbergSkala (MADRS) - die Depression mindestens ebenso gut lindern wie mit dem SSRI Sertralin. In der sechs Wochen dauernden Studie wurden 120 Patienten in vier Gruppen eingeteilt: Sie erhielten Sertralin (50 mg/d) oder Placebo in Kombination mit
tDCS oder Scheinstimulation. Die Kombi Placebo und Scheinstimulation besserte den MADRS-Wert um sechs Punkte, mit Sertralin und Scheinstimulation wurden neun und mit tDCS und Placebo elf Punkte erreicht. Die tDCS war damit sogar noch etwas wirksamer als die SSRI-Therapie, wenn auch nicht signifikant. Mit Abstand am wirksamsten war die Kombination beider Therapien: Erreicht wurde eine Besserung von 17,5 Punkten; damit hat man den Wert mehr als halbiert. Zudem wirkte die Therapie sehr schnell: Bereits nach zwei Wochen war der Wert um 15 Punkte gesunken. Ernsthafte Nebenwirkungen wurden nicht beobachtet. (mut)

Brunoni AR et al, JAMA Psychiatry 2013 (online first) 